\title{
Identification of monocyte-associated genes as predictive biomarkers of heart failure after acute myocardial infarction
}

\author{
Qixin Chen ${ }^{1 \dagger}$, Qijin Yinn ${ }^{2 \dagger}$, Junxian Song ${ }^{1 \dagger}$, Chuanfen Liu ${ }^{1}$, Hong Chen ${ }^{1 *}$ and Sufang Li ${ }^{1 *}$ (D)
}

\begin{abstract}
Background: Acute myocardial infarction (AMI) is a major contributor of heart failure (HF). Peripheral blood mononuclear cells (PBMCs), mainly monocytes, are the essential initiators of AMI-induced HF. The powerful biomarkers for early identification of AMI patients at risk of HF remain elusive. We aimed to identify monocyte-related critical genes as predictive biomarkers for post-AMI HF.

Methods: We performed weighted gene co-expression network analysis (WGCNA) on transcriptomics of PBMCs from AMI patients who developed HF or did not. Functional enrichment analysis of genes in significant modules was performed via Metascape. Then we obtained the single-cell RNA-sequencing data of recruited monocytes/ macrophages from AMl and control mice using the Scanpy and screened 381 differentially expressed genes (DEGs) between the two groups. We validated the expression changes of the 25 genes in cardiac macrophages from AMI mice based on bulk RNA-sequencing data and PBMCs data mentioned above.
\end{abstract}

Results: In our study, the results of WGCNA showed that two modules containing 827 hub genes were most significantly associated with post-AMI HF, which mainly participated in cell migration, inflammation, immunity, and apoptosis. There were 25 common genes between DEGs and hub genes, showing close relationship with inflammation and collagen metabolism. CUX1, CTSD and ADD3 exhibited consistent changes in three independent studies. Receiver operating characteristic curve analysis showed that each of the three genes had excellent performance in recognizing post-AMI HF patients.

Conclusion: Our findings provided a set of three monocyte-related biomarkers for the early prediction of HF development after AMl as well as potential therapeutic targets of post-AMI HF.

Keywords: Heart failure, Acute myocardial infarction, Biomarker, Systems biology, Monocyte

*Correspondence: chenhongbj@medmail.com.cn; Isf@bjmu.edu.cn ${ }^{\dagger}$ Qixin Chen, Qijin Yin and Junxian Song have contributed equally to this work

${ }^{1}$ Department of Cardiology, Beijing Key Laboratory of Early Prediction and Intervention of Acute Myocardial Infarction, Center for Cardiovascular Translational Research, Peking University People's Hospital, No 11. Xizhimen South Street, Xicheng District, Beijing 100044, China Full list of author information is available at the end of the article

\section{Background}

Heart failure (HF) is a growing global health problem affecting approximately 26 million people worldwide. It is estimated that more than 40 million people will develop this condition by 2030 [1]. HF has become a major cause of cardiovascular morbidity and mortality, which brought serious financial burden to both developed and developing countries $[2,3]$. Despite sufficient improvements in prevention and therapies, HF patients still present high hospitalization rates and poor survival rates $[4,5]$. 
Among different pathogenic factors, ST-segment-elevation myocardial infarction (STEMI), as the most common and severe type of acute myocardial infarction (AMI), is the major contributor of HF [6]. Thus, early recognition of AMI patients at risk of developing HF is an effective strategy for the reduction of HF incidence. Identifying early biomarkers associated with post-AMI HF may be helpful to resolve this issue. Though several biomarkers were proved to be related to HF triggered by AMI, such as natriuretic peptides (NPs), Galectin-3, soluble suppression of tumorigenicity-2 (sST2), growth/differential factor-15 (GDF-15) [7, 8], powerful biomarkers for early prediction of post-AMI HF remain elusive.

Cardiac remodeling, a major pathological change of HF, is characterized by cells apoptosis and necrosis, inflammation and immune cells activation, cardiomyocyte hypertrophy and myocardial interstitial fibrosis [9]. Increasing evidence indicated that $\sim 25 \%$ of survivors after STEMI developed HF due to cardiac remodeling [10-13]. PBMCs, mainly monocytes, are essential regulators of cardiac remodeling after AMI [9]. Monocytes rapidly move from bone marrow and spleen to blood and infiltrate the infarct zone and participate in the inflammatory response hours after myocardial injury $[14,15]$. Hence, genes involved in recruited monocytes/ macrophages-triggered cardiac remodeling may serve as promising biomarkers for the early identification of AMI patients at risk of HF development.

High-throughput microarray and transcriptome sequencing (such as bulk RNA- or single-cell RNAsequencing) permit us to perform a quick and comprehensive detection over the gene expression profiling, which are unbiased methods for screening diseasespecific biomarkers. Based on the transcriptomics data, several studies have identified potential biomarkers for prediction of HF following AMI via differentially expressed genes (DEGs) analysis [16-18], whereas which may result in the omission of some key genes related to disease. Weighted gene co-expression network analysis (WGCNA), as a bioinformatics application, can provide rich information based on calculating the pair-wise correlations between gene expression profiles [19]. WGCNA is thus increasingly used to identify key genes (termed hub genes) associated with specific disease or clinical trait [20-24].

Herein, we aimed to investigate monocyte-related critical genes as predictive biomarkers for HF development following AMI based on WGCNA. By using integrated analyses of gene expression profiles from microarray (samples: PBMCs from AMI patients), single-cell RNAsequencing (scRNA-seq, samples: recruited monocytes/ macrophages from AMI mice) and bulk RNA-sequencing (bulk RNA-seq, samples: cardiac macrophages from
AMI mice), we identified 3 key genes CUX1, CTSD, and ADD3 as potential biomarkers for early recognizing AMI patients at risk of developing HF.

\section{Methods \\ Data collection and processing}

The workflow of this study was shown in Fig. 1. The microarray gene expression data of PBMCs and scRNAseq data of cardiac macrophages were obtained from the Gene Expression Omnibus (GEO) database. The dataset GSE59867 from the Affymetrix Human Gene 1.0 ST Array [transcript (gene) version] [16], dataset GSE119355 from the $10 \times$ Genomics cell ranger platform [25] and published bulk RNA-seq data [26] were utilized in this study. In dataset GSE59867, the data of 17 PBMCs samples from AMI patients (within 1d after infarction) with or without HF development during a period of 6-month follow-up were analyzed (post-AMI HF, $n=9$ vs. post-AMI non-HF, $n=8$ ). The gene expression profiling was processed for background correction, $\log _{2}$ transformation and quantile normalization using the Robust Multiarray Average (RMA) algorithm. The array probes were mapped with respective gene symbol using the array annotations, and probes lacking annotation information were removed. Genes detected by more than one probe were counted only once. A total of 20,442 genes were included in the following analysis. For dataset GSE119355, the scRNA-seq data of 1806 cardiac macrophages from sham mice and 4697 macrophages from the ischemic area of myocardial infarction were processed using the Cell Ranger Single Cell software suite 1.3 .1 by $10 \times$ Genomics (http://10xgenomics.com/). In the validation data, cardiac macrophages of noninfarcted $(0 \mathrm{~d})$ or infarcted mice for $1 \mathrm{~d}, 3 \mathrm{~d}$, and $7 \mathrm{~d}$, isolated from 81 hearts including four biological replicates of $1.5 \times 10^{6}$ cells for each group, were analyzed using bulk RNA-seq. Then the whole transcriptome data were normalized using the method of fragments per kilobase of transcript per million mapped reads (FPKM).

\section{Weighted gene co-expression network analysis}

The 'WGCNA' R package was applied to identify diseaserelated modules and hub genes in these modules [19]. The 20,442 genes in dataset GSE59867 were filtered by median absolute deviation (MAD) to reduce noisy data. The top 10,000 highly variable genes were extracted for network analysis. The soft-threshold method of the Pearson correlation analysis was used to evaluate the possibility that two transcripts construct a weighted network. The appropriate soft-threshold power was screened based on the scale-free topology fit index of 0.8 , which was used to calculate the gene co-expression adjacency. The adjacency was transformed into Topological Overlap Matrix 


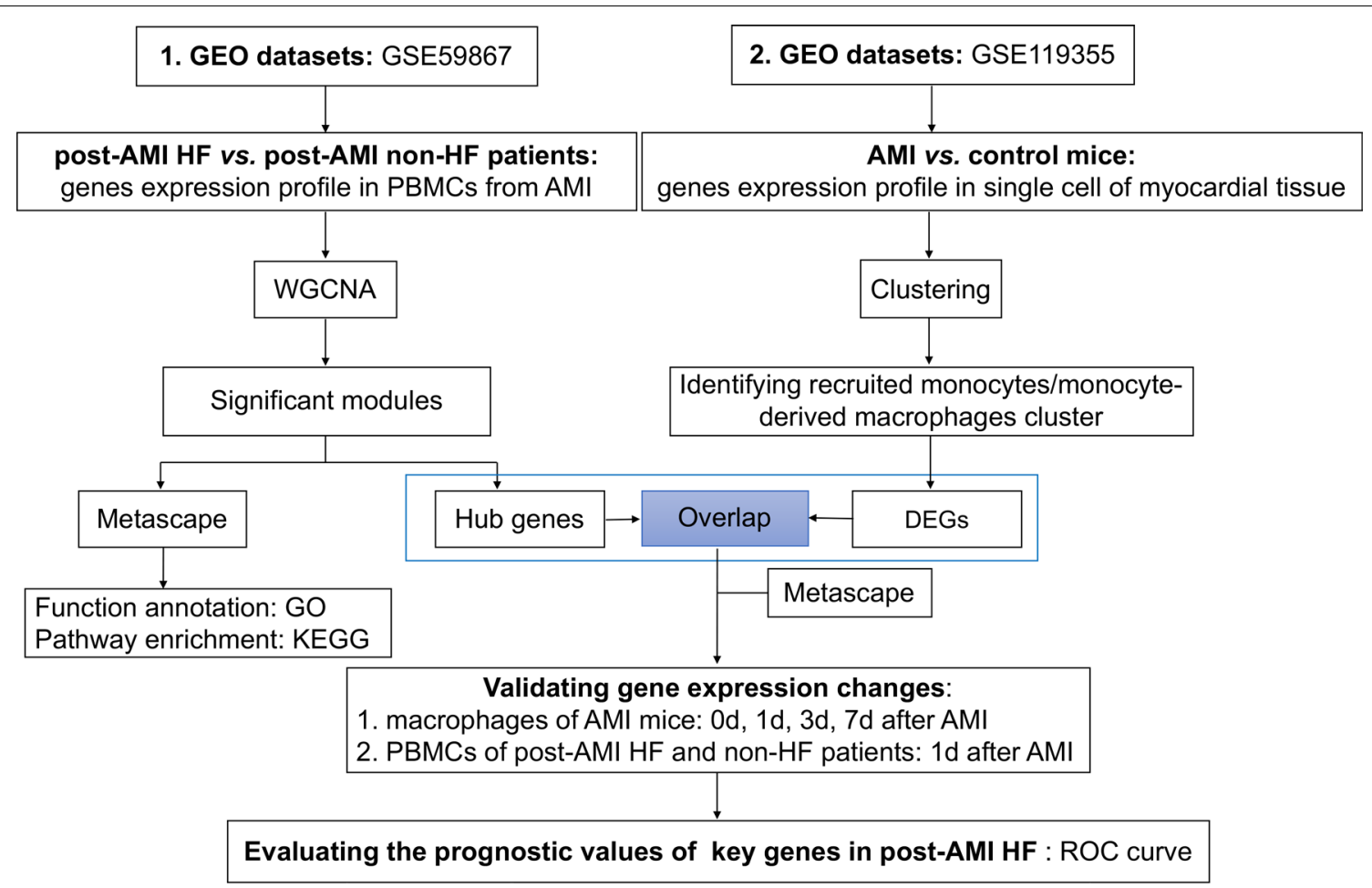

Fig. 1 Study flow chart. GEO Gene Expression Omnibus, AMI acute myocardial infarction, HF Heart Failure, PBMCs peripheral blood mononuclear cells, WGCNA Weighted Gene Co-expression Network Analysis, GO Gene Ontology, KEGG Kyoto Encyclopedia of Genes and Genomes, DEGs differentially expressed genes, $R O C$ receiver operating characteristic

(TOM) and the corresponding dissimilarity of genes co-expression (1-TOM) was calculated. Hierarchical clustering of the genes was conducted based on 1-TOM and shown with a clustering tree (dendrogram). Module identification was performed using the Dynamic Tree Cut method with minimal module size of 25 and module detection sensitivity (deepSplit) of 0.5 . The cut-off height of 0.3 , corresponding to correlation of 0.7 between the modules eigengenes, was chose to merge the similar modules. The correlation between module eigengenes (representing module) and post-AMI HF was calculated, and the modules with correlation coefficient $>0.5$ and $p$ value $<0.05$ were defined to be significantly related to post-AMI HF. Hub genes of each significant module were considered as those with gene significance $(|G S|)>0.35$ and module membership $(|\mathrm{MM}|)>0.8$, showing a significant correlation with the disease trait [27].

\section{scRNA-seq analysis}

The control and post-AMI samples were merged for the following analyses. The standard processing procedures of the dataset GSE119355, including filtering, highly variable genes identification, dimensionality reduction and clustering, were performed using the Scanpy [28]. The low-quality cells expressing $<200$ genes and genes expressed in $<3$ cells were filtered. To exclude the possible doublets, cells with $>5200$ genes were discarded. The poor-quality cells containing $>10 \%$ mitochondrial genes were also removed. Based on these criteria, 15,523 genes across 6357 cells in total remained for the subsequent analysis. The gene expression data underwent library-size normalization and log transformation. The highly variable genes with mean expression values between 0.0125 and 1.5 as well as minimal dispersion values of 0.5 were selected. Principal component analysis (PCA) was conducted on the highly variable genes for dimensionality reduction and 18 significant principal components were identified. Clustering in PCA space was performed using a graph-based clustering approach with a resolution of 0.5. The Louvain algorithm was applied for grouping cells into different clusters. Uniform Manifold Approximation and Projection (UMAP) was used for the two-dimensional visualization of the clustering results. The gene expression profile of recruited monocytes/monocytesderived macrophages cluster (446 cells) were extracted, and DEGs between control and post-AMI group were analyzed by Wilcoxon test. Genes with $\log _{2} \mathrm{FC}$ (fold change, FC, $>1.5$ ) $>0.585$ and adjusted p-value (pvals adj) $<0.05$ were considered as significantly DEGs. The top 10 DEGs in each group were visualized using a heatmap. 


\section{Functional enrichment analysis}

The genes of the significant modules were used for functional enrichment analysis. The Gene Ontology (GO) biological processes enrichment and Kyoto Encyclopedia of Genes and Genomes (KEGG) pathway analysis were performed via the Metascape [29]. $p$ value $<0.05$ in GO terms and KEGG pathways were considered statistically significant.

\section{Statistical analysis}

GraphPad Prism 8 was used for analyzing the gene expression level in bulk RNA-seq or microarray dataset. Data was shown as the mean \pm standard error of the mean (SEM). The normality of the data was verified by applying Shapiro-Wilk (S-W) normality test. Then the differences between two groups were compared via Mann-Whitney $U$ test for non-normally distributed data or unpaired two-tailed t-test for normally distributed data. Medcalc v19.1 was used for the receiver operator characteristic (ROC) curves analysis. The area under the ROC curve (AUC) was calculated to evaluate the specificity and sensitivity of single gene and their combination via binary logistic regression analysis. For all analyses, $p$ value $<0.05$ was considered statistically significant.

\section{Results}

\section{Construction of gene co-expression network and identification of modules}

Seventeen PBMCs samples of AMI patients on day 1 after infarction containing 20,442 genes were included, and the top 10,000 highly variable genes were used for the co-expression network construction. When the scalefree fit index reached 0.8 , the lowest soft-thresholding power 22 was selected to generate hierarchical clustering of the 10,000 genes (Additional file 1: Figure S1A).

The hierarchical clustering of the genes was analyzed based on 1-TOM. The identified 15 modules and the correlations between genes expression in each module and post-AMI HF were displayed in Additional file 1: Figure S1B (Additional file 5: Table S3) (red, positive correlation; blue, negative correlation). The gray module (insignificant module) containing unassigned genes was discarded.

The co-expression similarity of the 15 modules was quantified by calculating the correlations between module eigengenes. The clustering dendrogram showed that the 15 modules were divided into two main clusters (Additional file 2: Figure S2A), which was also confirmed by the heatmap of module eigengenes adjacencies (Additional file 2: Figure S2B).

\section{Identification of modules significantly correlated} with post-AMI HF and corresponding hub genes

The results of module-trait relationship analysis showed that 3 of the 15 modules, turquoise, blue and midnightblue module were significantly correlated with post-AMI HF (Fig. 2a). Specifically, the turquoise module was positively associated with post-AMI HF (correlation coefficient $=0.537, p<0.05$ ), whereas the blue (correlation coefficient $=-0.545, p<0.05)$ and midnightblue modules (correlation coefficient $=-0.516, p<0.05$ ) were negatively associated with the disease. The turquoise, blue and midnightblue modules separately contained 2814, 2115 and 31 genes (Additional file 6: Table S4).

The $\log _{10}$ transformation of $\mathrm{p}$ value in the linear regression between gene expression and post-AMI HF was used to represent GS. MM was determined as the average absolute GS for all genes in a module. Here, the GS and $\mathrm{MM}$ in the turquoise, blue and midnightblue modules were shown as scatterplots (Fig. 2b). Highly significant correlations between GS and MM were only observed in turquoise (correlation coefficient $=0.4, p<0.05$ ) and blue modules (correlation coefficient $=-0.4, p<0.05$ ). Based on the cut-off criteria of $|\mathrm{MM}|>0.8$ and $|\mathrm{GS}|>0.35,382$ and 445 highly connected hub genes separately corresponding to the turquoise and blue modules were identified (Additional file 7: Table S5).

\section{Functional enrichment analysis of genes in significant modules}

To understand the function of genes in turquoise and blue modules, GO enrichment and KEGG pathway analysis were performed via the Metascape platform. 2814 genes of turquoise module and 2115 genes of blue module were separately uploaded to the platform. The results of biological process in $\mathrm{GO}$ enrichment were exhibited in Fig. 3a, b (Additional file 2: Table S1). Genes in turquoise module were enriched in the AMI-related pathological process, such as myeloid leukocyte activation, regulation of cell adhesion, regulation of cytokine production, positive regulation of cell migration, regulation of inflammatory response, and positive regulation of cell death. Genes in blue module were mainly concentrated in the immunity, apoptosis and cell cycle, such as lymphocyte differentiation, antigen receptor-mediated signaling pathway, intrinsic apoptotic signaling pathway by p53 class mediator, regulation of cell cycle $\mathrm{G} 2 / \mathrm{M}$ phase transition. The results of KEGG pathway analysis indicated that genes in turquoise modules were mainly involved in Notch signaling pathway, Chemokine signaling pathway, MAPK signaling pathway, TNF signaling pathway, and genes in blue modules were mainly implicated in Cell cycle, Primary immunodeficiency, Antigen processing 


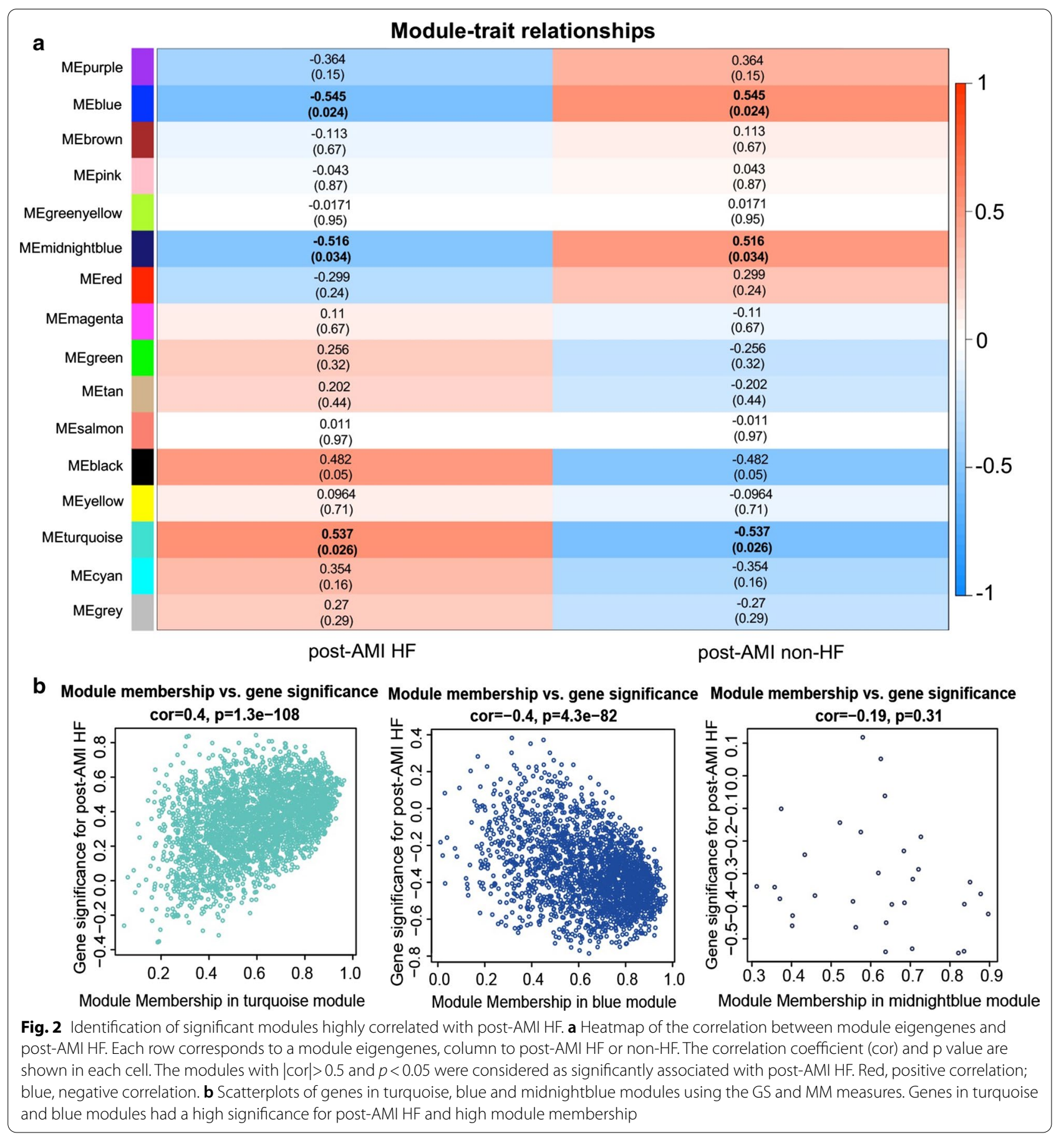

and presentation, and Th17 cell differentiation (Fig. 3c, d, Additional file 4: Table S2).

\section{Analysis of differentially expressed genes of recruited monocytes/macrophages in infarcted cardiac tissue} In the early stage of AMI, the composition of cardiac macrophages changes dramatically. Circulating pro-inflammatory monocytes $(\mathrm{CCR} 2+)$ rapidly infiltrate infarcted myocardium within hours, then differentiate into CCR2+ macrophages to promote inflammatory responses, disorders of collagen metabolism, and ultimately contribute to HF pathogenesis [30]. In order to investigate whether some of the hub genes screened above were involved in the regulatory role of recruited 


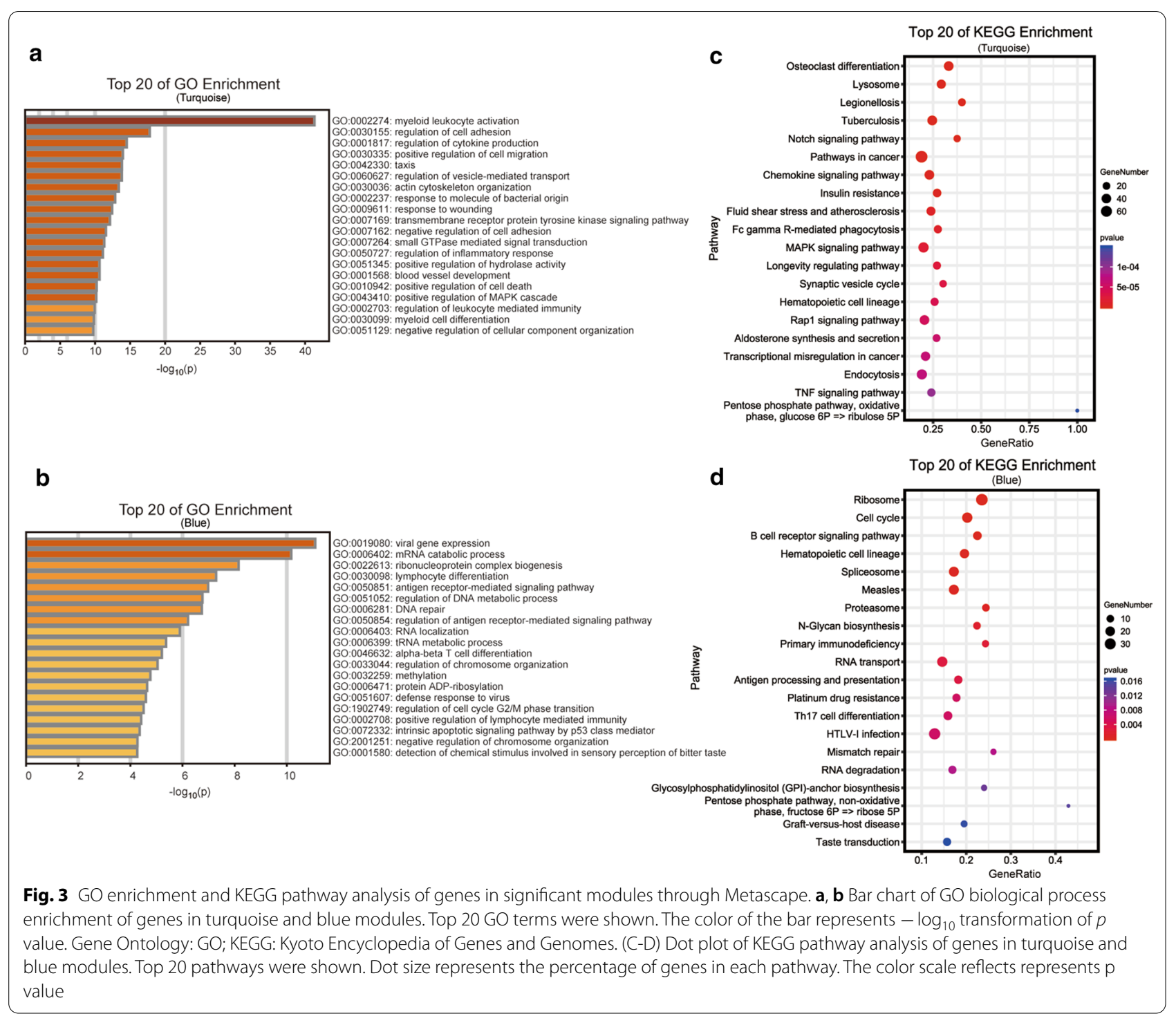

monocytes/macrophages in HF development, based on the scRNA-seq data in GSE119355, we identified CCR2+ monocytes/macrophages subset in infarcted cardiac tissue of mice, and obtained the gene expression profiling of each single cell. UMAP analysis for all populations of the cardiac macrophages in both infarcted and non-infarcted mice identified 9 clusters, among which the circulating monocytes derived CCR2+ monocytes/macrophages cluster (macrophages marker: Ccr2, Plac8; monocytes marker: Ace) were focused on this study (Fig. 4a, c). The distribution of all cardiac macrophages from infarcted and non-infarcted mice were shown in Fig. 4b, indicating an obvious increase of macrophages.

Subsequently, we compared the genes expression profiling of CCR2+ monocytes/macrophages cluster in infarcted and non-infarcted mice. Results demonstrated that 103 genes were upregulated, and 278 genes were downregulated in AMI mice compared with controls (Additional file 8: Table S6). The top 20 DEGs (10 upregulated and 10 downregulated) were exhibited in Fig. $4 \mathrm{~d}$.

Furthermore, we found 25 common genes between the 381 DGEs and the 827 hub genes (Fig. 5a). GO biological process enrichment showed that the 25 genes were enriched in myeloid leukocyte activation, collagen metabolic process and response to hypoxia, suggesting a close relationship with cardiac remodeling (Fig. 5b). These 25 genes might be involved in the regulatory role of circulating monocytes in AMI-triggered HF and were selected for the subsequent validation analysis.

Validation of expression levels of common genes in cardiac macrophages from AMI mice and PBMCs from AMI patients To determine the reliable biomarkers for the prediction of post-AMI HF, the expression levels of 25 common 
a

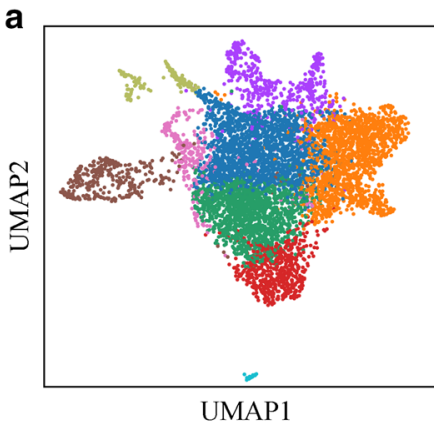

- 0_Mhc-Il cluster

- 1_macrophages-1 cluster

- 2_macrophages-2 cluster

- 3_Timd4 cluster

- 4_CCR2 + monocytes/macrophages

5_proliferating macrophages cluster - 6_Isg cluster

7 Cd209a cDC2s cluster

- 8_macrophages-3 cluster

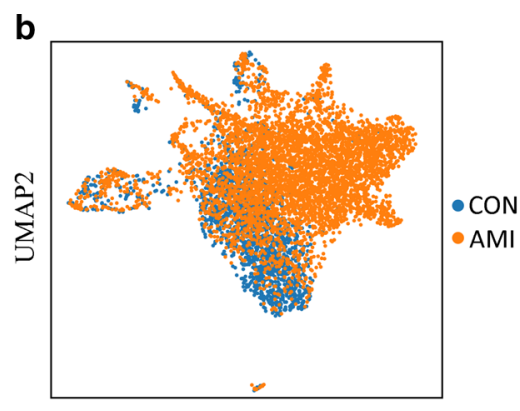

UMAP1

C

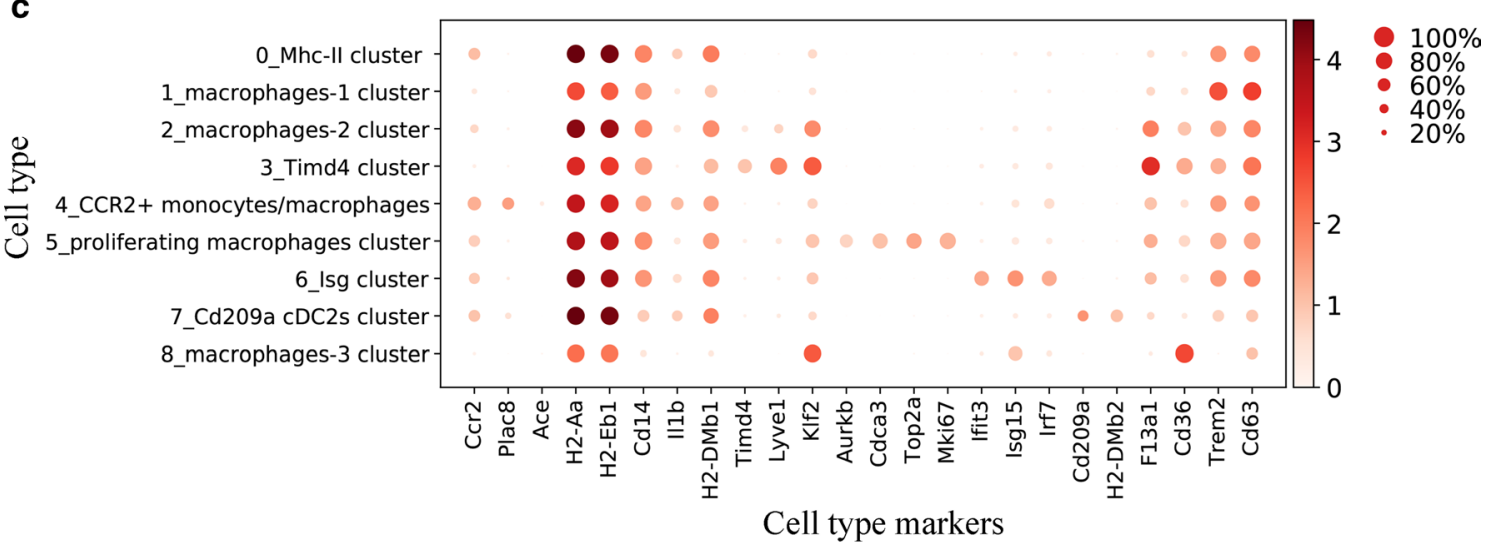

d

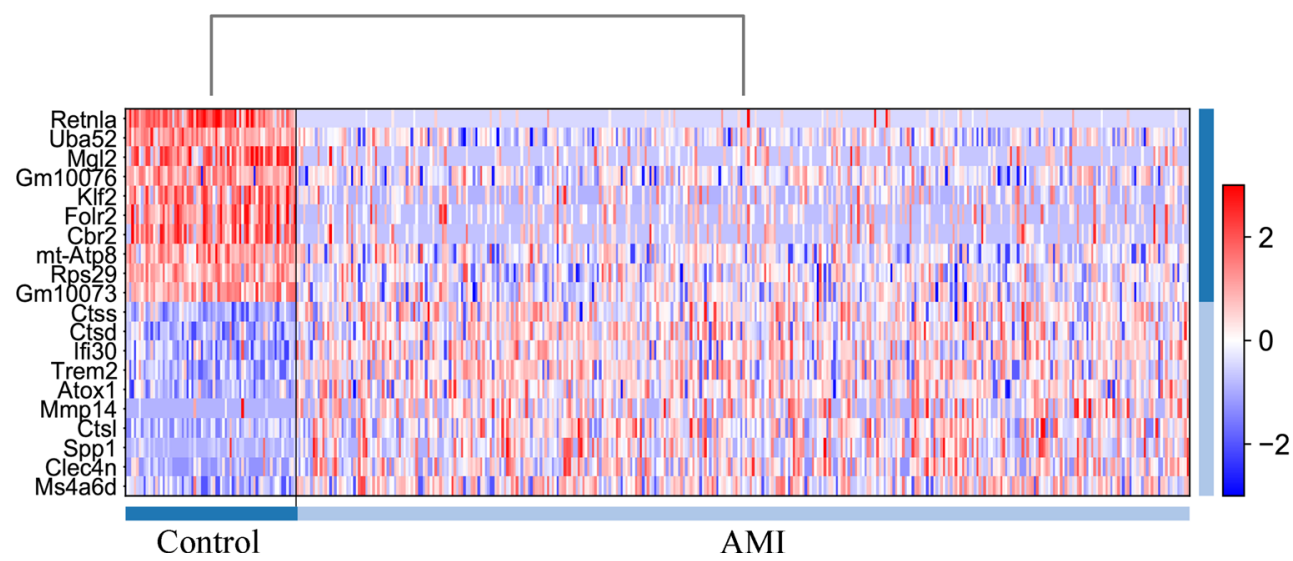

Fig. 4 Identifying recruited monocytes/monocytes-derived macrophages cluster (CCR2+ monocytes/macrophages) in the infarcted cardiac tissue of mice. a UMAP visualization of cell clusters present in pooled control and post-AMI samples. CCR2+ monocytes/macrophages were identified as cluster 4. b UMAP plot depicting all cell types in control (blue) and MI samples (yellow). c Dot plot of marker genes for each macrophage cluster. Dot size represents the percentage of cells expressing the marker gene in each cell cluster. The color scale reflects the gene expression level from low to high. $\mathbf{d}$ Heatmap of top 20 differently expressed genes (10 upregulated and 10 downregulated genes) in CCR2+ monocytes/macrophages between AMl and control mice. The color scale indicates the gene expression level (blue: low; red: high). UMAP: Uniform Manifold Approximation and Projection

genes were firstly validated in the bulk RNA-seq data of cardiac macrophages from mice before and on days 1,3 and 7 post AMI [26]. We observed that 7 out of 25 genes showed the consistent changes with those in the scRNA-seq data, including 4 upregulated genes (MMP14,
CUX1, CTSD, PKM) and 3 downregulated genes (ADD3, ALOX5, RCN3) (Fig. 6a). Additionally, the expression levels of the 7 genes were verified in the microarray data of GSE59867 which contained 9 PBMCs samples from AMI patients with HF development and 8 with no HF 


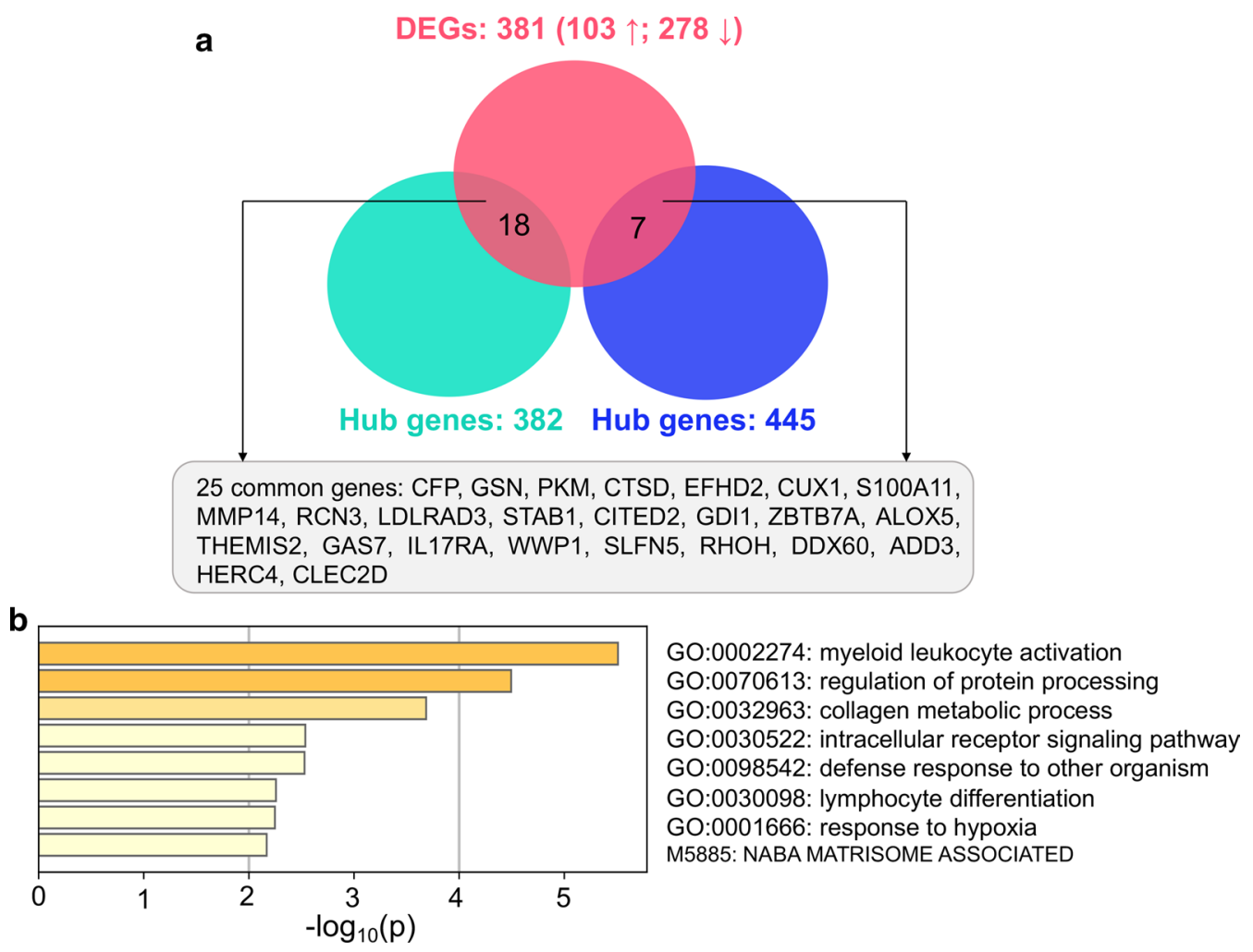

Fig. 5 GO biological process enrichment of common genes between DEGs and hub genes. a Overlap between DEGs and hub genes of turquoise and blue modules. A total of 25 common genes were identified. b The GO biological process enrichment of 25 common genes through Metascape. DEGs differentially expressed genes, GO Gene Ontology

development. The results showed consistent increase in levels of CUX1 and CTSD and consistent decrease in levels of ADD3 (Fig. 6b). Thus, CUX1, CTSD and ADD3 were ultimately selected for the following ROC curves analysis.

\section{Effectiveness evaluation of validated genes as prognostic biomarkers of AMI with risk of $\mathrm{HF}$}

To evaluate the power of CUX1, CTSD and ADD3 as predictive biomarkers of HF after AMI, ROC curves analysis was constructed and the AUCs were calculated based on the dataset GSE59867 mentioned above. The corresponding AUCs of CTSD, CUX1 and ADD3 were 0.889 (95\% CI 0.644-0.987; $p=0.0001$ ), 0.861 (95\% CI $0.610-0.960$; $p=0.0015)$ and 0.819 (95\% CI 0.0.561-0.960; $p=0.0023$ ), respectively (Fig. 7, Table 1 ). The combinations of CTSD and CUX1 had the highest AUC $(0.917$; CI 0.680-0.995, $p<0.0001$ ) (Fig. 7, Table 1), showing the best specificity and sensitivity in identifying AMI patients at risk of HF progression.

\section{Discussion}

In the present study, based upon the gene expression profiling of PBMCs from patients suffered STEMI for 1 day who developed HF or did not during a 6-month follow-up, we identified modules significantly correlated with post-AMI HF as well as hub genes in these modules using WGCNA. Subsequently, we obtained the DEGs of recruited monocytes/macrophages cluster in infarct area of AMI mice. The expression levels of common genes between the hub genes and DEGs were further validated both in cardiac macrophages of another independent group of AMI mice and in PBMCs of AMI patients mentioned above. Consistently upregulated CUX1, CTSD and downregulated ADD3 in these 3 independent studies were identified, and were proved to be potential biomarkers for the early prediction of post-AMI HF. Moreover, the 3 genes might be involved in the regulation of recruited monocytes/macrophages in cardiac remodeling after AMI, and hence serve as potential therapeutic targets of post-AMI HF.

The primarily pathological characteristics of cardiac remodeling during HF development after AMI include inflammatory and immune response, oxidative stress, 
(See figure on next page.)

Fig. 6 Validation of common genes expression in cardiac macrophages from AMI mice and PBMC from AMI patients. a The gene expression changes in cardiac macrophages over the AMI time course, before (day 0) and on days 1, 3 and 7 post AMl, detected by RNA-seq. Seven out of 25 common genes exhibited consistent changes with that in the recruited monocytes/macrophages from infarcted myocardium were shown. ${ }^{*} p<0.05,{ }^{* *} p<0.01$ compared with group AMI-d0. b The expression levels of 7 common genes in PBMC from AMl patients at admission measured by microarray. ${ }^{*} p<0.05 ;{ }^{* *} p<0.01$ compared with group post-AMI non-HF; n.s.: no significance. post-AMl non-HF group: $\mathrm{n}=8$, post-AMl HF group: $\mathrm{n}=9$. Data are presented as mean \pm SEM

mitochondrial dysfunction, apoptosis, cardiomyocyte hypertrophy, degradation of the extracellular matrix and fibrosis $[8,9]$. In line with these characteristics, functional enrichment analysis in our study showed that genes in HF-related modules mainly participated in inflammatory/inflammation-associated response, immune response, apoptosis and cell cycle transition. The corresponding pathways were Notch signaling pathway, chemokine signaling pathway, MAPK signaling pathway, TNF signaling pathway, cell cycle, antigen processing and presentation, Th17 cell differentiation, DNA replication, all of which play an importantly regulatory role in the cardiac remodeling and consequent $\operatorname{HF}[8,9,31,32]$. These results suggested that AMI patients with developing HF might have more serious inflammation, immunity and apoptosis in infarct zones than whom without HF.

Recruited monocytes/monocyte-derived macrophages in myocardium are key regulators in the AMI-induced cardiac remodeling. The recruited macrophages in infracted myocardium were usually classified into proinflammatory (1-4 day post AMI) and anti-inflammatory phenotype (5 days post AMI) [33]. However, growing evidence has indicated that the canonical M1/M2 phenotypes of monocyte-derived macrophages can no longer present all the macrophages subpopulations in infarcted myocardium [34-36]. Defining macrophages only with M1/M2 subtypes may produce misleading conclusions [36]. The high-throughput scRNA-seq can identify unbiased clustering of cells based on the transcriptome analysis at single-cell level, which overcomes the drawback of simple definition of macrophage phenotypes. Therefore, to accurately clarify the relationship between circulating monocytes/monocyte-derived macrophages and post-AMI HF, we obtained the gene expression profiling of the recruited monocytes/macrophages cluster based on the scRNA-seq data of cardiac macrophages from AMI and control mice [25]. Among the 381 DEGs in recruited monocytes/macrophages of infarcted mice, 25 genes overlapped with the hub genes in PBMCs from AMI patients. The functional enrichment analysis of the 25 common genes manifested that they were involved in proinflammatory leukocyte activation, hypoxia response and collagen metabolic process. These results collectively demonstrated that recruited monocytes/macrophages may modulate cardiac remodeling and HF after AMI through these altered genes.

To further determine the reliability of candidate genes as biomarkers for the early recognition of AMI patients at risk of HF, we verified the expression levels of the 25 common genes in cardiac macrophages of mice before and after AMI for $1 \mathrm{~d}, 3 \mathrm{~d}$ and $7 \mathrm{~d}$. There were 7 consistently changed genes in scRNA-seq dataset. There was an overlapping period of recruited macrophages exhibiting pro-inflammatory and anti-inflammatory activity during the first 7 days post AMI [35], which offered an explanation of why each of these 7 genes kept the consistent changes at the different phases of AMI. Among the 7 genes validated in PBMCs from patients at 1 day of AMI (GSE59867), CUX1, CTSD and ADD3 had the similar expression changes to those in the recruited macrophages of AMI mice. A recent study revealed that transcription factor CUX1, a known tumor suppressor [37], is a key regulator of inflammation in rheumatoid arthritis. CUX1 coupled with $\mathrm{I} \mathrm{B} \zeta$ mediated neutrophil and monocyte recruitment by increasing the production of multiple chemokines and cytokines [38], suggesting an adverse effect of CUX1 on cardiac remodeling after AMI. CTSD, a major lysosomal protease, is increasingly known for its involvement in inflammatory response, and has been proposed as biomarkers of several inflammatory diseases, such as non-alcoholic steatohepatitis [39], atherosclerosis and coronary heart disease [40]. CTSD might contribute to plaque vulnerability by inducing macrophages apoptosis [41] and high levels of plasma CTSD was associated with increased risk of future coronary syndromes [42]. Similarly, in dataset GSE59867, CTSD mRNA level in PBMC of post-AMI HF patients at different time points after AMI was higher than that in post-AMI non-HF patients (data not shown). However, the results were inconsistent with that in another study, which indicated that serum CTSD activity decreased in AMI patients with new-onset HF during 6-month follow-up compared to patients without post-AMI HF [43]. Hence, larger clinical trial based on uniform specimen source (PBMCs, plasma or serum) and detecting item (mRNA, protein or protein activity) are necessary to clarify the real relationship between CTSD and postAMI HF. ADD3, a structural cytoskeletal protein [44], has been implicated to participate in the monocytes 


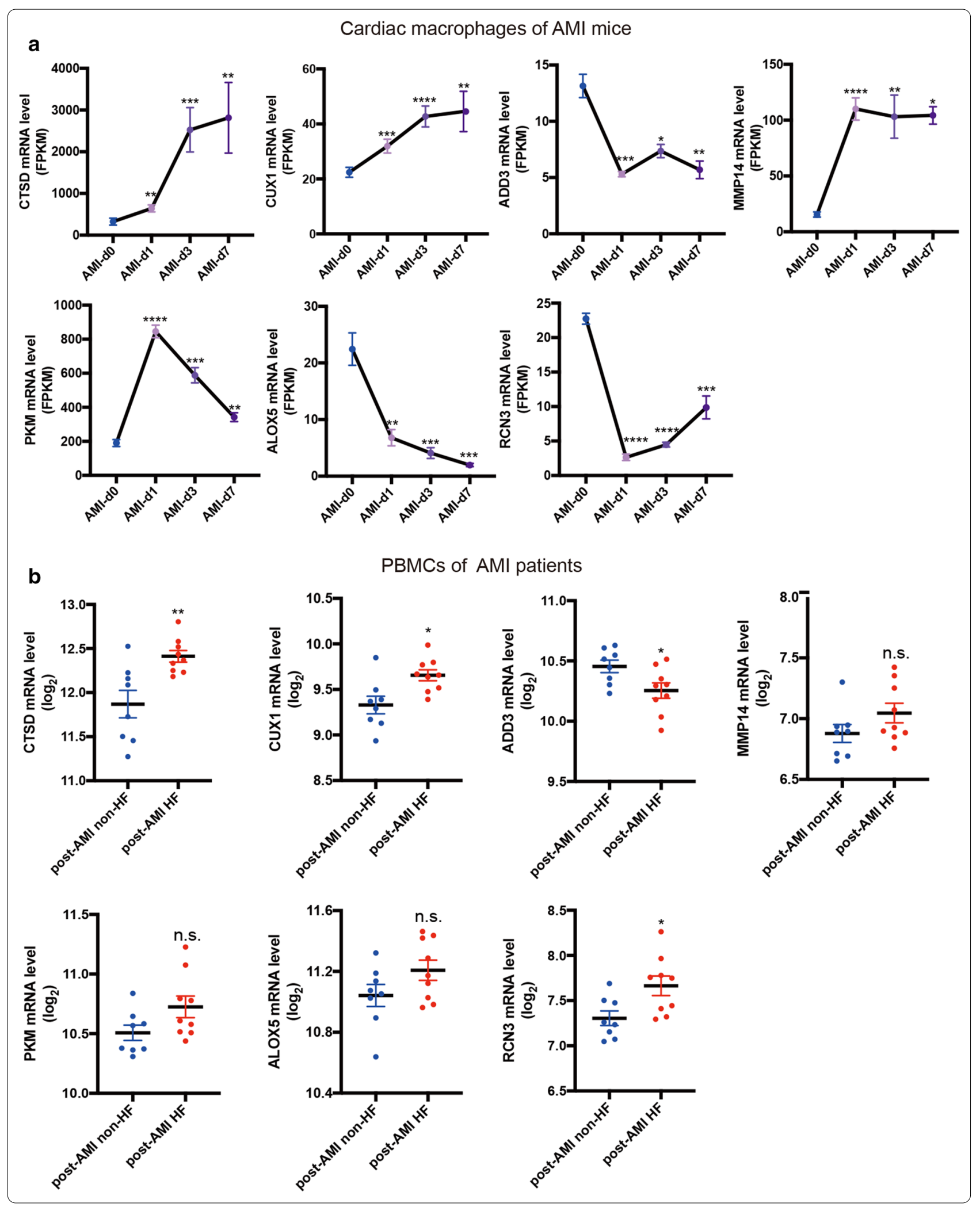




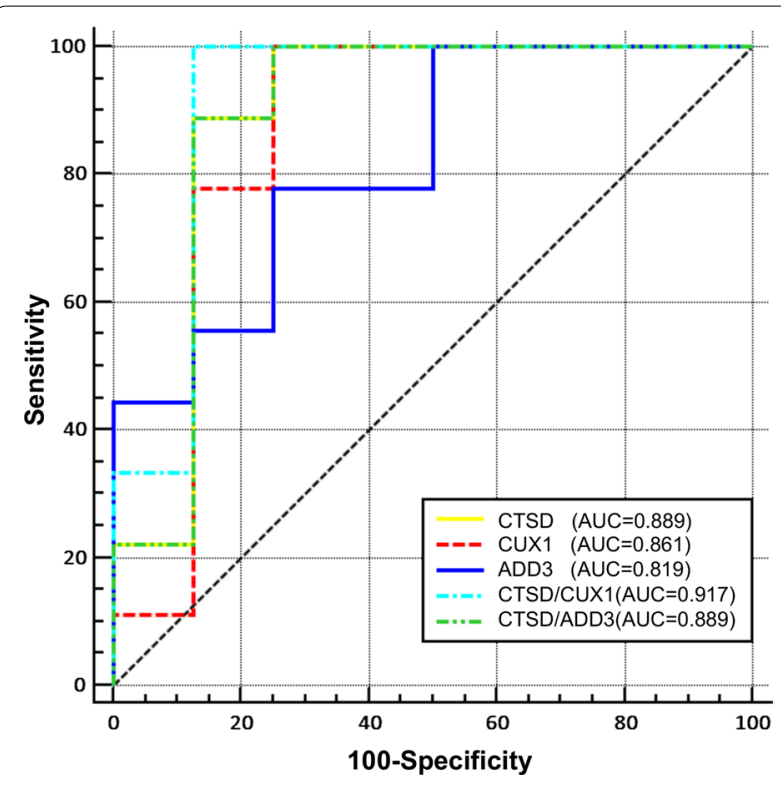

Fig. 7 Receiver operator characteristic (ROC) curves analysis of potential biomarkers. The areas under the ROC curves (AUCs) were given for single gene (CUX1, CTSD and ADD3) and combinations (CTSD/CUX1, CTSD/ADD3) to recognize AMI patients who developed HF during a 6-month followed-up. post-AMI non-HF group: $n=8$, post-AMI HF group: $n=9$. ROC curves were constructed using the $\log _{2}$ transformed expression data of GSE59867

\section{Conclusions}

In this study, we constructed the gene co-expression network of PBMCs from AMI patients who developed HF or did not during a 6-month follow-up using WGCNA, and identified significant modules correlated with post-AMI HF. Three hub genes of the significant modules CUX1, CTSD and ADD3 showed the consistent expression changes in PBMCs of AMI patients and recruited monocytes/macrophages of AMI mice, which may mediate the circulating monocytes-triggered cardiac remodeling and HF development. Monocyte-related CUX1, CTSD and ADD3 are promising biomarkers for early identifying AMI patients at risk of HF and potential therapeutic targets of post-AMI HF. Large clinical trials are needed to further validate the predictive value of the 3 genes as biomarkers of HF development after AMI.

\section{Supplementary information}

The online version contains supplementary material available at https://doi. org/10.1186/s12920-021-00890-6.

Additional file 1: Figure $\mathbf{S 1}$. Identification of modules through WGCNA. (A) Analysis of scale-free fit index (left panel) and mean connectivity (right panel) for various soft-thresholding powers. The lowest soft-thresholding power was 22 when the scale-free fit index reached 0.8, (B) Clustering dendrogram of genes, with dissimilarity based on TOM, together with the original module colors (Dynamic Tree Cut) and assigned merged module colors (Module colors), as well as the heatmap of correlations between

Table 1 Receiver operating characteristic curves

\begin{tabular}{lllc|rrr|}
\hline & AUC & 95\% Cl & $\boldsymbol{p}$ value & Cut-off value & Specificity (\%) & Sensitivity (\%) \\
\hline CTSD & 0.889 & $0.644-0.987$ & 0.0001 & $>12.2266$ & 87.50 & 88.89 \\
CUX1 & 0.861 & $0.610-0.960$ & 0.0015 & $>9.3887$ & 75.00 & 100.00 \\
ADD3 & 0.819 & $0.561-0.960$ & 0.0023 & $\leq 10.3521$ & 75.00 & 77.78 \\
CTSD/CUX1 & 0.917 & $0.680-0.995$ & $<0.0001$ & $>0.0102$ & 87.50 & 100.00 \\
CTSD/ADD3 & 0.889 & $0.644-0.987$ & $<0.0001$ & $>1.1740$ & 87.50 & 88.89 \\
\hline
\end{tabular}

AUC area under the curve, $\mathrm{Cl}$ confidence interval

migration as well as monocyte-to-macrophage differentiation [45]. The genetic variation of ADD3 was also reported to be associated with left ventricular diastolic relaxation [46]. These findings implied the potential modulation of ADD3 in HF development after AMI.

In the ROC curves analysis, an AUC of 0.7 to 0.8 is regarded acceptable, 0.8 to 0.9 is excellent, and more than 0.9 is outstanding [47]. The AUC values of CUX1, CTSD and ADD3 were all more than 0.8 , showing high diagnostic accuracy for identifying AMI patients at risk of HF. In particular, the AUC value of combinations of CTSD and CUX1 reached 0.917 , exhibiting outstanding capability to identify the target patients. genes expression and post-AMI HF. Under the condition of soft-thresholding power of 22, minimal module size of 25 and cut height of $0.3,15$ modules were identified. A short vertical line in clustering dendrogram corresponds to a gene and highly co-expressed genes are grouped together. Blue: negative correlation; red: positive correlation.

Additional file 2: Figure S2. The clustering dendrogram of modules eigengenes and heatmap of the correlations between modules eigengenes. (A) Hierarchical clustering of module eigengenes. Module eigengenes in each branch of the dendrogram were highly positively correlated. (B) Heatmap of the adjacencies in the modules eigengenes network. The modules eigengenes correlations represent modules similarities. Each column and row correspond to one module eigengenes. Red means high adjacency (positive correlation) of two modules, while blue means low adjacency (negative correlation). Red squares along the diagonal are the meta-module.

Additional file 3: Table S1. GO biological processes in co-expression modules. 
Additional file 4: Table S2. KEGG pathways in co-expression modules

Additional file 5: Table S3. Gene_trait_correlation_p-value.

Additional file 6: Table S4. Genes of significant modules.

Additional file 7: Table S5. Hub genes of turquoise and blue modules.

Additional file 8: Table S6. DEGs of AMI vs. Con.

\section{Abbreviations}

AMI: Acute myocardial infarction; HF: Heart failure; PBMCs: Peripheral blood mononuclear cells; DEGs: Differentially expressed genes; STEMI: ST-segmentelevation myocardial infarction; NPs: Natriuretic peptides; SST2: Soluble suppression of tumorigenicity-2; GDF-15: Growth/differential factor-15; WGCNA: Weighted gene co-expression network analysis; scRNA-seq: Single-cell RNA-sequencing; Bulk RNA-seq: Bulk RNA-sequencing; GEO: Gene Expression Omnibus; RMA: Robust Multiarray Average; FPKM: Fragments per kilobase of transcript per million mapped reads; MAD: Median absolute deviation; TOM: Topological overlap Matrix; GS: Gene significance; MM: Module membership; cor: Correlation coefficient; PCA: Principal component analysis; UMAP: Uniform Manifold Approximation and Projection; pvals_adj: Adjusted p-value; GO: Gene Ontology; KEGG: Kyoto Encyclopedia of Genes and Genomes; SEM: Standard error of the mean; ROC: Receiver operator characteristic curves; AUC: Area under the ROC curve; CCR2+: Circulating pro-inflammatory monocytes.

\section{Acknowledgements}

Not applicable.

\section{Authors' contribution}

$\mathrm{HC}$ and SFL designed and supervised the study and revised the manuscript. QXC, QJY and JXS analyzed the data and drafted the manuscript. CFL searched literatures and made interpretations of the data. All authors read and approved the final manuscript.

\section{Funding}

This research was funded by the National Natural Science Foundation of China (Nos. 81770356, 81970301, 82070519, 81600340), Beijing Municipal Natural Science Foundation (No.7202218) and the Capital Health Research and Development of Special (No. 2020-2-4084). The funding body provided support in collection, analysis and interpretation of data and in writing the manuscript.

\section{Availability of data and materials}

All data generated or analyzed during this study are included in this published article and its supplementary Additional files. The datasets analyzed during the current study are available in the NCBI-Gene Expression Omnibus (GEO) public repository, [https://www.ncbi.nlm.nih.gov/geo/query/acc. cgi?acc=gse59867] and [https://www.ncbi.nlm.nih.gov/geo/query/acc. cgi?acc=GSE1 19355]. GEO accession numbers for microarray gene expression data of PBMCs and sCRNA-seq data of cardiac macrophages are GSE59867 and GSE119355, respectively.

\section{Ethics approval and consent to participate}

Not applicable.

\section{Consent for publication}

Not applicable.

\section{Competing interests}

The authors declare that they have no competing interests.

\section{Author details}

${ }^{1}$ Department of Cardiology, Beijing Key Laboratory of Early Prediction and Intervention of Acute Myocardial Infarction, Center for Cardiovascular Translational Research, Peking University People's Hospital, No 11. Xizhimen South Street, Xicheng District, Beijing 100044, China. ${ }^{2}$ Ministry of Education Key Laboratory of Bioinformatics, Research Department of Bioinformatics at the Beijing National Research Center for Information Science and Technology, Center for Synthetic and Systems Biology, Department of Automation, Tsinghua University, Beijing 100084, China.
Received: 18 October 2020 Accepted: 31 January 2021

Published online: 09 February 2021

\section{References}

1. Chen X, Savarese G, Dahlström U, Lund LH, Fu M. Age-dependent differences in clinical phenotype and prognosis in heart failure with mid-range ejection compared with heart failure with reduced or preserved ejection fraction. Clin Res Cardiol. 2019;108(12):1394-405.

2. Carter HE, Schofield D, Shrestha R. Productivity costs of cardiovascular disease mortality across disease types and socioeconomic groups. Open Heart. 2019;6(1):e000939.

3. Yap J, Chia SY, Lim FY, Allen JC, Teo L, Sim D, et al. The Singapore heart failure risk score: prediction of survival in Southeast Asian patients. Ann Acad Med Singap. 2019;48(3):86-94.

4. Anderson JL, Morrow DA. Acute myocardial infarction. N Engl J Med. 2017;376(21):2053-64.

5. Giustino G, Redfors B, Brener SJ, Kirtane AJ, Généreux P, Maehara A, et al Correlates and prognostic impact of new-onset heart failure after STsegment elevation myocardial infarction treated with primary percutaneous coronary intervention: insights from the INFUSE-AMI trial. Eur Heart J Acute Cardiovasc Care. 2018;7(4):339-47.

6. Vernon ST, Coffey S, D'Souza M, Chow CK, Kilian J, Hyun K, et al. ST-segment-elevation myocardial infarction (STEMI) patients without standard modifiable cardiovascular risk factors-how common are they, and what are their outcomes? J Am Heart Assoc. 2019;8(21):e013296.

7. Feistritzer HJ, Klug G, Reinstadler SJ, Reindl M, Mayr A, Mair J, et al. Novel biomarkers predicting cardiac function after acute myocardial infarction. Br Med Bull. 2016;119(1):63-74.

8. Berezin AE, Berezin AA. Adverse cardiac remodelling after acute myocardial infarction: old and new biomarkers. Dis Markers. 2020;2020:1215802.

9. Zimmer A, Bagchi AK, Vinayak K, Bello-Klein A, Singal PK. Innate immune response in the pathogenesis of heart failure in survivors of myocardial infarction. Am J Physiol Heart Circ Physiol. 2019:316(3):H435-45.

10. Kelly DJ, Gershlick T, Witzenbichler B, Guagliumi G, Fahy M, Dangas G, et al. Incidence and predictors of heart failure following percutaneous coronary intervention in ST-segment elevation myocardial infarction: the HORIZONS-AMI trial. Am Heart J. 2011;162(4):663-70.

11. Caccioppo A, Franchin L, Grosso A, Angelini F, D'Ascenzo F, Brizzi MF. Ischemia reperfusion injury: mechanisms of damage/protection and novel strategies for cardiac recovery/regeneration. Int J Mol Sci. 2019;20(20):5024

12. Danchin N, Popovic B, Puymirat E, Goldstein P, Belle L, Cayla G, et al. Fiveyear outcomes following timely primary percutaneous intervention, late primary percutaneous intervention, or a pharmaco-invasive strategy in ST-segment elevation myocardial infarction: the FAST-MI programme. Eur Heart J. 2020;41(7):858-66.

13. Thrane PG, Kristensen SD, Olesen KKW, Mortensen LS, Bøtker HE, Thuesen $L$, et al. 16-year follow-up of the Danish Acute Myocardial Infarction 2 (DANAMI-2) trial: primary percutaneous coronary intervention vs. fibrinolysis in ST-segment elevation myocardial infarction. Eur Heart J. 2020;41(7):847-54

14. Nahrendorf M, Swirski FK, Aikawa E, Stangenberg L, Wurdinger T, Figueiredo $\mathrm{J}$, et al. The healing myocardium sequentially mobilizes two monocyte subsets with divergent and complementary functions. J Exp Med. 2007;204(12):3037-47.

15. Sager HB, Kessler T, Schunkert H. Monocytes and macrophages in cardiac injury and repair. J Thorac Dis. 2017;9(Suppl 1):S30-5.

16. Maciejak A, Kiliszek M, Michalak M, Tulacz D, Opolski G, Matlak K, et al. Gene expression profiling reveals potential prognostic biomarkers associated with the progression of heart failure. Genome Med. 2015;7(1):26.

17. Li X, Li B, Jiang H. Identification of time-series differentially expressed genes and pathways associated with heart failure post-myocardial infarction using integrated bioinformatics analysis. Mol Med Rep. 2019;19(6):5281-90.

18. Azuaje F, Devaux Y, Wagner DR. Coordinated modular functionality and prognostic potential of a heart failure biomarker-driven interaction network. BMC Syst Biol. 2010;4:60.

19. Langfelder P, Horvath S. WGCNA: an R package for weighted correlation network analysis. BMC Bioinform. 2008;9:559. 
20. Chen J, Yu L, Zhang S, Chen X. Network analysis-based approach for exploring the potential diagnostic biomarkers of acute myocardial infarction. Front Physiol. 2016;7:615

21. Gandal MJ, Haney JR, Parikshak NN, Leppa V, Ramaswami G, Hartl C, et al. Shared molecular neuropathology across major psychiatric disorders parallels polygenic overlap. Science. 2018;359(6376):693-7.

22. Chen R, Ge T, Jiang W, Huo J, Chang Q, Geng J, et al. Identification of biomarkers correlated with hypertrophic cardiomyopathy with coexpression analysis. J Cell Physiol. 2019;234(12):21999-2008.

23. Song ZY, Chao F, Zhuo Z, Ma Z, Li W, Chen G. Identification of hub genes in prostate cancer using robust rank aggregation and weighted gene co-expression network analysis. Aging (Albany NY). 2019;11(13):4736-56.

24. Chen $P$, Long $B, X u Y$, Wu W, Zhang S. Identification of crucial genes and pathways in human arrhythmogenic right ventricular cardiomyopathy by coexpression analysis. Front Physiol. 2018;9:1778.

25. Dick SA, Macklin JA, Nejat S, Momen A, Clemente-Casares X, Althagafi $M G$, et al. Self-renewing resident cardiac macrophages limit adverse remodeling following myocardial infarction. Nat Immunol. 2019;20(1):29-39.

26. Mouton AJ, DeLeon-Pennell KY, Rivera Gonzalez OJ, Flynn ER, Freeman TC, Saucerman JJ, et al. Mapping macrophage polarization over the myocardial infarction time continuum. Basic Res Cardiol. 2018;113(4):26.

27. Tang J, Kong D, Cui Q, Wang K, Zhang D, Gong Y, et al. Prognostic genes of breast cancer identified by gene co-expression network analysis. Front Oncol. 2018:8:374.

28. Wolf FA, Angerer P, Theis FJ. SCANPY: large-scale single-cell gene expression data analysis. Genome Biol. 2018;19(1):15.

29. Zhou Y, Zhou B, Pache L, Chang M, Khodabakhshi AH, Tanaseichuk O, et al. Metascape provides a biologist-oriented resource for the analysis of systems-level datasets. Nat Commun. 2019;10(1):1523.

30. Lavine KJ, Epelman S, Uchida K, Weber KJ, Nichols CG, Schilling JD, et al. Distinct macrophage lineages contribute to disparate patterns of cardiac recovery and remodeling in the neonatal and adult heart. Proc Natl Acad Sci USA. 2014;111(45):16029-34.

31. Nemir M, Metrich M, Plaisance I, Lepore M, Cruchet S, Berthonneche C, et al. The Notch pathway controls fibrotic and regenerative repair in the adult heart. Eur Heart J. 2014;35(32):2174-85.

32. He Y, Pang S, Huang J, Zhu K, Tong J, Tang Y, et al. Blockade of RBP-Jmediated notch signaling pathway exacerbates cardiac remodeling after infarction by increasing apoptosis in mice. Biomed Res Int 2018;2018:5207031.

33. Ma Y, Mouton AJ, Lindsey ML. Cardiac macrophage biology in the steadystate heart, the aging heart, and following myocardial infarction. Transl Res. 2018;191:15-28.

34. Nahrendorf M, Swirski FK. Abandoning M1/M2 for a network model of macrophage function. Circ Res. 2016;119(3):414-7.
35. Walter W, Alonso-Herranz L, Trappetti V, Crespo I, Ibberson M, Cedenilla $M$, et al. Deciphering the dynamic transcriptional and post-transcriptional networks of macrophages in the healthy heart and after myocardial injury. Cell Rep. 2018;23(2):622-36.

36. Peet C, Ivetic A, Bromage DI, Shah AM. Cardiac monocytes and macrophages after myocardial infarction. Cardiovasc Res. 2020;116(6):1101-12

37. Liu N, Sun Q, Wan L, Wang X, Feng Y, Luo J, et al. CUX1, a controversial player in tumor development. Front Oncol. 2020;10:738.

38. Slowikowski K, Nguyen HN, Noss EH, Simmons DP, Mizoguchi F, Watts GFM, et al. CUX1 and IKBZ (NFKBIZ) mediate the synergistic inflammatory response to TNF and IL-17A in stromal fibroblasts. Proc Natl Acad Sci USA. 2020;117(10):5532-41.

39. Houben T, Oligschlaeger Y, Hendrikx T, Bitorina AV, Walenbergh SMA, van Gorp PJ, et al. Cathepsin D regulates lipid metabolism in murine steatohepatitis. Sci Rep. 2017;7(1):3494.

40. Mohammadpour AH, Salehinejad Z, Elyasi S, Mouhebati M, Mirhafez SR, Samadi S, et al. Evaluation of serum cathepsin D concentrations in coronary artery disease. Indian Heart J. 2018;70(4):471-5.

41. Li W, Yuan XM. Increased expression and translocation of lysosomal cathepsins contribute to macrophage apoptosis in atherogenesis. Ann NY Acad Sci. 2004;1030:427-33.

42. Gonçalves I, Hultman K, Dunér P, Edsfeldt A, Hedblad B, Fredrikson GN, et al. High levels of cathepsin D and cystatin B are associated with increased risk of coronary events. Open Heart. 2016;3(1):e000353.

43. Yamac AH, Sevgili E, Kucukbuzcu S, Nasifov M, Ismailoglu Z, Kilic E, et al. Role of cathepsin D activation in major adverse cardiovascular events and new-onset heart failure after STEMI. Herz. 2015;40(6):912-20.

44. Matsuoka Y, Li X, Bennett $\mathrm{V}$. Adducin: structure, function and regulation. Cell Mol Life Sci. 2000;57(6):884-95.

45. de Bruin RG, Shiue L, Prins J, de Boer HC, Singh A, Fagg WS, et al. Quaking promotes monocyte differentiation into pro-atherogenic macrophages by controlling pre-mRNA splicing and gene expression. Nat Commun. 2016;7:10846.

46. Kuznetsova T, Citterio L, Herbots L, Carpini SD, Thijs L, Casamassima N, et al. Effects of genetic variation in adducin on left ventricular diastolic function as assessed by tissue Doppler imaging in a Flemish population. $J$ Hypertens. 2008;26(6):1229-36.

47. Mandrekar JN. Receiver operating characteristic curve in diagnostic test assessment. J Thorac Oncol. 2010:5(9):1315-6.

\section{Publisher's Note}

Springer Nature remains neutral with regard to jurisdictional claims in published maps and institutional affiliations.
Ready to submit your research? Choose BMC and benefit from:

- fast, convenient online submission

- thorough peer review by experienced researchers in your field

- rapid publication on acceptance

- support for research data, including large and complex data types

- gold Open Access which fosters wider collaboration and increased citations

- maximum visibility for your research: over 100M website views per year

At BMC, research is always in progress.

Learn more biomedcentral.com/submissions 